\title{
The safety profile of lumbar spinal surgery in elderly patients 85 years and older
}

\author{
Michael Y. Wang, MD, Gabriel Widi, MD, and Allan D. Levi, MD, PhD \\ Department of Neurological Surgery, University of Miami Miller School of Medicine, Miami, Florida
}

OBJECT The aging of the population will require that surgeons increasingly consider operating on elderly patients. Performing surgery safely in the elderly will require an understanding of the factors that predict successful outcomes and avoid complications.

METHODS Records of patients 85 years and older undergoing elective lumbar spinal surgery were retrospectively reviewed. Microdiscectomies were excluded. Preexisting medical illnesses measured using the Charlson Comorbidity Index (CCI), American Society of Anesthesiologists (ASA) Physical Status class, age, and surgical parameters were analyzed as factors potentially predictive of complications. Ambulatory function was rated on a 4-point scale.

RESULTS During the study 26 consecutive patients (mean age 87 years) with a mean ASA class of $2.6 \pm 0.65$ and $\mathrm{CCl}$ of $1.1 \pm 1.27$ were enrolled. The average number of levels treated was $2.17 \pm 1.23$, and $73 \%$ underwent fusion. The mean follow-up was 41.9 months with a minimum of 24 months, and all patients were alive at last follow-up. Average blood loss was $142 \pm 184 \mathrm{ml}$, and the operative time was $183.3 \pm 80.6$ minutes. The mean number of levels treated was $2.17 \pm 1.13$ (range 1-4). Ambulatory function improved significantly by $0.59 \pm 1.0$ points. Five complications (19.2\%) occurred in 4 patients, 2 major and 3 minor. Four complications were temporary and 1 was permanent. Patient age, blood loss, $\mathrm{CCl}$ score, ASA class, the number of levels treated, and fusion surgery were not statistically associated with a complication. Operative time of longer than 180 minutes $(p=0.0134)$ was associated with complications.

CONCLUSIONS Lumbar spine surgery in patients 85 years and older can be accomplished safely if careful attention is paid to preoperative selection. Prolonged operative times are associated with a higher risk of complications.

http://thejns.org/doi/abs/10.3171/2015.7.FOCUS15180

KEY WORDS elderly; spine; complications; lumbar spine; fusion

$\Delta$ $s$ the US population ages, the prevalence of degenerative spinal conditions will increase markedly. It is now estimated that the population 65 years and older will increase by $92.5 \%$ from 41.4 million in 2011 to 79.7 million in 2040. With this demographic change will come an increased risk of perioperative complications, posing a challenge for spinal surgeons. The risks of anesthetic complications, surgical complications, and postoperative adverse events can all be increased with comorbidities that are associated with advancing age.

Because age is commonly perceived as an independent risk factor for complications with spinal surgery, many surgeons are reluctant to treat patients of advanced age. While the specific age at which any surgical procedure is considered too dangerous is highly variable between surgeons and anesthesiologists, this determination is ultimately subjective. The perception of age as a contributor to surgical risk has also changed over time. Several de- cades ago a patient older than 75 years would likely have been considered too old for any surgical intervention, yet now surgery is routinely performed in patients in this age group.

Numerous studies have demonstrated that the surgical treatment of lumbar degenerative disease can produce profound improvements in function in properly selected patients. When successful, patients can experience dramatic improvements in pain, ambulation, and activities of daily living. In addition, the prevalence of degenerative conditions such as spinal stenosis, arthritis of the intervertebral disc or facet joints, and spinal deformities increase as a patient ages, potentially creating a conundrum of increased rates of pathology in patients who are at higher risk for treatment. $1,2,7,8,11$ Indeed, in a recent review of Medicare claims data for 2002-2007, the rate of complex fusion procedures increased from 1.3 to 19.9 per 100,000 beneficiaries. ${ }^{5}$ 
We reviewed our recent experience in patients who would universally be considered to be at high risk for surgery given their age alone. Utilizing a cutoff of 85 years of age or older, we were interested in determining the incidence, scope, and severity of perioperative complications. Furthermore, this study sought to identify any prognostic factors or maneuvers to be used in the management of these challenging patients.

\section{Methods}

We retrospectively reviewed cases of degenerative lumbar spine surgery performed in patients 85 years of age or older by 2 spine neurosurgeons at a single tertiary academic medical center. Twenty-six consecutive cases were identified over a 76-month period. Patient evaluation consisted of standing upright flexion/extension radiographs, CT scanning, and MRI. Their clinical symptoms were then correlated to these findings to determine the need and extent of surgical intervention. Surgical candidates consisted of patients in whom conservative therapy had failed, including physical therapy, injections, and medical management or those who presented with a neurological deficit with imaging concordant with the physical examination. All patients exhibited significant pain and disability due to radiculopathy, neurogenic claudication, axial pain due to lumbar degeneration, or mechanical instability. The preoperative medical condition of these patients was reviewed using the weighted Charlson Comorbidity Index (CCI) (Table 1), ${ }^{4}$ and the American Society of Anesthesiologists (ASA) Physical Status classification (http://www. asahq.org/resources/clinical-information/asa-physicalstatus-classification-system), in which Class I signifies a normal healthy patient; Class II, a patient with mild systemic disease; Class III, a patient with severe systemic disease; Class IV, a patient w/ severe systemic disease that is a constant threat to life; Class V, a moribund patient who is not expected to survive without the operation; and Class VI, a declared brain-dead patient whose organs are being removed for donor purposes. All surgically treated patients underwent preoperative clearance by their primary medical physician as well as by a cardiologist and any other medical specialists as needed.

Perioperative complications in these cases were identified retrospectively using chart review. Complications were classified based on the system affected (neurologicalspine, neurological-central, wound, integumentary, cardiac, pulmonary, electrolyte, hematological, thrombotic, skeletal, and death); severity (minor vs major); and temporal extent (temporary vs permanent). Patients undergoing a unilateral microdiscectomy were excluded due to the minimal nature of these interventions. The operative interventions had to include either bilateral lumbar decompression or an instrumented fusion.

Ambulatory function was rated on a 4-point scale (4, independently ambulatory; 3 , requiring a cane; 2 , requiring a walker; and 1, wheelchair bound). Intraoperative blood loss calculations were obtained from the anesthesia record for each surgery. Patient age, operative time, blood loss, CCI score, ASA class, the number of treated levels in cases of spinal pathology, and the inclusion of a fusion were analyzed as potential predictive factors for periop-
TABLE 1. Summary of the $\mathrm{CCl}$

\begin{tabular}{lc}
\hline \multicolumn{1}{c}{ Comorbidity } & Relative Weight Assignment \\
\hline Metastatic solid tumor & 6 \\
\hline AIDS & 6 \\
\hline Moderate-to-severe liver disease & 3 \\
\hline Hemiplegia & 2 \\
\hline Moderate-to-severe renal failure & 2 \\
\hline Diabetes w/ end organ damage & 2 \\
\hline Neoplasia & 2 \\
\hline Leukemia/lymphoma & 2 \\
\hline Myocardial infarct & 1 \\
\hline Congestive heart failure & 1 \\
\hline Peripheral vascular disease & 1 \\
\hline Cerebrovascular disease & 1 \\
\hline Dementia & 1 \\
\hline Chronic pulmonary disease & 1 \\
\hline Connective tissue disease & 1 \\
\hline Ulcer disease & 1 \\
\hline Mild liver disease & 1 \\
\hline Diabetes & 1 \\
\hline
\end{tabular}

* The total score is obtained by adding the relative weight of each comorbidity.

erative complications and improvements in the ambulatory score immediately following surgery and at 3 months postoperatively using the Student t-test or chi-square test. These assessments were performed by chart review and not the treating surgeons. Due to the sample size limitations variables were also analyzed by partitioning ordinally as follows: patient age ( $<90$ years vs $\geq 90$ years), operative time $(<180$ minutes vs $\geq 180$ minutes $)$, blood loss $(<500 \mathrm{ml}$ vs $\geq 500 \mathrm{ml})$, CCI score ( $\leq 2 \mathrm{vs}>2)$, ASA class ( $\leq$ II vs $>$ II), number of treated levels $(\leq 2$ vs $>2$ ) in cases of spinal pathology, and the inclusion of a fusion.

\section{Results}

During the study period 26 consecutive patients 85 years and older were treated with lumbar spinal surgery for degenerative conditions. The population characteristics are presented in Table 2. The mean age was $87 \pm$ 2.1 years (range 85-93), and 16 of the patients were male. The mean ASA class was $2.6 \pm 0.65$ (range I-III). The mean CCI was $1.1 \pm 1.27$ (range $0-8$ ). Of these, 19 surgeries (73\%) involved a fusion, and 12 would be considered minimally invasive (46\%). While 7 of the patients were former smokers, none of the patients were smoking at the time of surgery. The mean number of levels treated was $2.17 \pm 1.23$ (range 1-5). The mean follow-up was 41.9 months following surgery, with a minimum of 24 months. All patients were alive at last follow-up.

The mean operative blood loss was $142 \pm 184 \mathrm{ml}$ (range $10-750 \mathrm{ml}$ ). The total operative time averaged $183.3 \pm 80.6$ minutes (range 61-342 minutes). Ambulatory function improved significantly in this cohort, with mean scores improving from $2.68 \pm 1.06$ to $3.15 \pm 0.92$, representing a mean improvement of $0.59 \pm 1.0$. Twelve $(46 \%)$ of the patients experienced ambulatory improvement, and 3 of 
TABLE 2. Patient profiles*

\begin{tabular}{lcc}
\hline \multicolumn{1}{c}{ Variable } & Value & Range \\
\hline Mean age in yrs & $87 \pm 2.1$ & $85-93$ \\
\hline Male/female, no. & $16: 10$ & \\
\hline Mean ASA class & $2.6 \pm 0.65$ & I-III \\
\hline Mean combined CCI & $1.1 \pm 1.27$ & $0-8$ \\
\hline Mean no. of treated levels & $2.17 \pm 1.13$ & $1-5$ \\
\hline Mean op time in mins & $183 \pm 80.6$ & $61-342$ \\
\hline Mean surgical blood loss in $\mathrm{ml}$ & $142 \pm 184$ & $10-750$ \\
\hline Minimally invasive surgery & $46 \%$ & \\
\hline Fusion surgery & $73 \%$ & \\
\hline Mean ambulatory score & & \\
\hline Preop & $2.68 \pm 1.06$ & \\
\hline Postop & $3.15 \pm 0.92$ \\
\hline Change & $0.59 \pm 1.0$ & \\
\hline Mean values arepresented as the &
\end{tabular}

* Mean values are presented as the mean \pm SD.

these had 2 points of improvement. One patient worsened by 1 point.

In this cohort a total of 5 complications (19.2\%) occurred in 4 patients (Table 3). There was a single complication in each of the categories of neurological-spine worsening, integumentary, and pulmonary; there were 2 electrolyte complications. Two complications would be classified as major, and 3 as minor. Four were temporary and 1 was permanent. One 88-year-old patient who underwent an L5S1 interbody fusion for a spondylolisthesis suffered from hyponatremia and developed a decubitus ulcer. Another 88-year-old patient who underwent a laminectomy had worsening proximal leg weakness, resulting in decreased reduced ambulatory function. An 85-year-old patient who underwent a 4-level fusion for a spinal deformity developed postoperative pneumonia and had to be reintubated, ultimately recovering. Finally, an 88-year-old patient with polymyalgia rheumatic disease who underwent a lumbar decompression developed postoperative hyponatremia, prolonging his hospital stay. There were no deaths.

An analysis of predictive factors for any complication showed that patient age, blood loss, CCI score, ASA class, the number of levels treated, and fusion surgery were not statistically associated with a complication. However, operative time of greater than 180 minutes $(p=0.0134)$ was associated with complications. The average time of surgery for patients with a complication was 196.8 minutes versus 176.9 minutes in those without a complication.

\section{Discussion}

Elective spinal surgery carries with it specific challenges and opportunities. Because procedures can be timed to allow for medical optimization prior to an intervention, the surgeon can reduce the likelihood of complications through optimal cardiovascular care, nutritional support, smoking cessation, and physical preconditioning. However, the elective nature of these surgeries also suggests that the operative intervention can be delayed indefinitely if the risk is perceived to be too high. Prognostication of risk is, of course, an imperfect science and is subjective,
TABLE 3. Complications

\begin{tabular}{lc}
\hline \multicolumn{1}{c}{ System } & No. of Patients (\%) \\
\hline Neurological-spine & 1 \\
\hline Neurological-central & 0 \\
\hline Wound & 0 \\
\hline Integumentary & 1 \\
\hline Cardiac & 0 \\
\hline Pulmonary & 1 \\
\hline Electrolyte & 2 \\
\hline Hematological & 0 \\
\hline Thrombotic & 0 \\
\hline Skeletal & 0 \\
\hline Death & 0 \\
\hline Severity & \\
\hline Major & $2(40)$ \\
\hline Minor & $3(60)$ \\
\hline Temporal extent & \\
\hline Temporary & $4(80)$ \\
\hline Permanent & $1(20)$ \\
\hline
\end{tabular}

incorporating the input of the surgeon, anesthesiologist, and other managing physicians.

It is frequently perceived that advancing age independently increases the risk of a surgical intervention. In a study by Fineberg et al., the Nationwide Inpatient Sample from 2002 to 2009 was used to determine predictive factors for cardiac complications with spinal surgery. ${ }^{6}$ That study found that in 578,457 lumbar spine procedures the incidence of cardiac complications was 6.7 of 1000 cases. These events were more frequent following fusion than nonfusion operations (9.3 of 1000 cases vs 4.0 of 1000 cases). Patients suffering a cardiac complication were 9.4 years older than those without complications. ${ }^{6}$

However, the role that age plays in surgical and anesthesia risk is highly variable among patients, as other physiological parameters are likely to be a greater predictor of surgical safety. While elective spinal surgery may not be classified as life-saving, withholding spinal surgery to treat structural pathologies such as neural compression or segmental instability may have significant implications for the patient. Restrictions in mobility, dependency on narcotic medications, and persistent pain can have major implications for not only an elderly patient's well-being, but also for survival. Physical deconditioning in frail patients can impact life expectancy due to progressive pulmonary and cardiac complications.

In a previous study, we examined patient outcomes following lumbar spinal stenosis surgery in 88 patients older than 75 years of age. ${ }^{10}$ Preexisting medical conditions were analyzed using the CCI, and pre- and postoperative ambulatory function was rated. Our findings indicated that the only significant factors predictive of wound and systemic complications were length of operative time $(p=0.003)$ and the CCI $(\mathrm{p}=0.088)$. Thus, lumbar spine surgery in a select population of elderly patients may be safe if the CCI is acceptable and operative times are minimized. 
Since our previous study, several other authors have examined spinal surgery-related risks in the elderly. Carreon et al. conducted a retrospective study of 98 patients older than 65 years of age who required lumbar decompression and arthrodesis in older adults and determined that the complication rate correlated with older age, increased blood loss, longer operative time, and number of levels of arthrodesis. ${ }^{3}$ In another large study using data from the Veterans Affairs National Surgical Quality Improvement Program patients who underwent cervical corpectomy were studied $(n=1560)$. Multivariate analysis showed that age older than 80 years had an odds ratio of 21.24 for predicting complications. Type 1 diabetes, ASA class greater than III, dependent functional status, 3 or more corpectomy levels, and operative duration longer than 6 hours were also predictors of complications.

A similar study by Raffo and Lauerman retrospectively studied 20 patients older than 80 years who were treated for lumbar spine disorders and found that comorbidities, hospital stay, and intensive care unit stay were the only factors that correlated with inpatient outcomes. ${ }^{9}$ Thus, there is evidence emphasizing the importance of evaluating the general medical health of the patient independent of age while considering surgical intervention.

In this study we found that complication rates were higher (19.2\%) than generally reported in diverse age groups. However, only 2 major complications occurred, and there were no perioperative deaths. We did find that increased operative times were associated with complications, but this study was underpowered to determine the effects of comorbidities and ASA class to predict complications. An important caveat is that these elderly patients were carefully preselected and reflect the very tight indications and considerations of medical comorbidities in our preoperative planning.

Due to the large population of elderly patients treated in our practice, we have adopted several strategies empirically in an attempt to minimize perioperative complications. In all cases in which substantial blood loss is expected, we use the cell saver for autotransfusion. Maintenance of normal blood pressure is also essential for perfusion of vital structures, and decreases in blood pressure rarely have a significant beneficial effect on surgical blood loss. In all cases careful coordination with the anesthesiologist is obviously essential. In addition, it is essential to consider the patient's life expectancy in any discussions regarding surgery for improving quality of life. Those with long life expectancies remaining, independent of their current age, are the most likely to enjoy the benefits of surgery and the complications of continued disabling spinal problems.

The clear limitations to this study are the limited sample size and lack of a contemporaneous comparator group of either younger patients or patients of the same age treated conservatively. Large series of patients in this age category are difficult to accrue, and registries or multiinstitutional databases typically lack granular data that allow for detailed analysis of specific variables and outcomes. Furthermore, this study did not encompass the myriad patients 85 years and older who refused or were declined operative intervention.

\section{Conclusions}

Spinal surgery to treat lumbar degenerative disease can be safe in the extremely elderly patient. The rate of complications is higher than with younger patients but can be acceptable when balanced against the potential benefits of the intervention. Careful attention to the patients' comorbidities is critical, and anesthetic and operative times must be kept to a minimum.

\section{References}

1. Acosta FL Jr, McClendon J Jr, O'Shaughnessy BA, Koller H, Neal CJ, Meier O, et al: Morbidity and mortality after spinal deformity surgery in patients 75 years and older: complications and predictive factors. J Neurosurg Spine 15:667-674, 2011

2. Benz RJ, Ibrahim ZG, Afshar P, Garfin SR: Predicting complications in elderly patients undergoing lumbar decompression. Clin Orthop Relat Res (384):116-121, 2001

3. Carreon LY, Puno RM, Dimar JR II, Glassman SD, Johnson JR: Perioperative complications of posterior lumbar decompression and arthrodesis in older adults. J Bone Joint Surg Am 85-A:2089-2092, 2003

4. Charlson ME, Pompei P, Ales KL, MacKenzie CR: A new method of classifying prognostic comorbidity in longitudinal studies: development and validation. J Chronic Dis 40:373383, 1987

5. Deyo RA, Mirza SK, Martin BI, Kreuter W, Goodman DC, Jarvik JG: Trends, major medical complications, and charges associated with surgery for lumbar spinal stenosis in older adults. JAMA 303:1259-1265, 2010

6. Fineberg SJ, Ahmadinia K, Patel AA, Oglesby M, Singh K: Incidence and mortality of cardiac events in lumbar spine surgery. Spine (Phila Pa 1976) 38:1422-1429, 2013

7. Lehto MU, Honkanen P: Factors influencing the outcome of operative treatment for lumbar spinal stenosis. Acta Neurochir (Wien) 137:25-28, 1995

8. Quigley MR, Kortyna R, Goodwin C, Maroon JC: Lumbar surgery in the elderly. Neurosurgery 30:672-674, 1992

9. Raffo CS, Lauerman WC: Predicting morbidity and mortality of lumbar spine arthrodesis in patients in their ninth decade. Spine (Phila Pa 1976) 31:99-103, 2006

10. Wang MY, Green BA, Shah S, Vanni S, Levi ADO: Complications associated with lumbar stenosis surgery in patients older than 75 years of age. Neurosurg Focus 14(2): e7, 2003

11. Zheng F, Sandhu HS, Cammisa FP Jr, Girardi FP, Khan SN: Predictors of functional outcome in elderly patients undergoing posterior lumbar spine surgery. J Spinal Disord 14:518521,2001

\section{Disclosure}

Dr. Wang reports that he is a consultant and patent holder with DePuy Spine, is a consultant for Aesculap Spine, receives royalties from Quality Medical Publishing, and receives support of non-study-related clinical or research effort from Department of Defense. Dr. Levi reports that he receives educational honoraria from Medtronic Sofamor Danek and DePuy Synthes.

\section{Author Contributions}

Conception and design: Wang. Acquisition of data: Wang, Widi. Analysis and interpretation of data: Wang, Widi. Drafting the article: Wang. Critically revising the article: Wang, Levi.

\section{Correspondence}

Michael Y. Wang, Department of Neurological Surgery, University of Miami, 1095 N.W. 14th Terrace, Lois Pope LIFE Center, Miami, FL 33136. email: mwang2@med.miami.edu. 